\title{
Persistent currents of superfluidic light in a four-level coherent atomic medium
}

\author{
Nuno A. Silva, ${ }^{1,2, *}$ (i) J. T. Mendonça, ${ }^{3}$ And A. Guerreiro ${ }^{1,2}$ \\ ${ }^{1}$ Departamento de Física e Astronomia da Faculdade de Ciências da Universidade do Porto, Rua do Campo Alegre 687, 4169-007 Porto, Portugal \\ 2INESC TEC, Centre of Applied Photonics, Rua do Campo Alegre 687, 4169-007 Porto, Portugal \\ ${ }^{3}$ IPFN, Instituto Superior Técnico Universidade de Lisboa, 1049-001 Lisboa, Portugal \\ ${ }^{*}$ Corresponding author: nunoazevedosilva@gmail.com
}

Received 8 June 2017; revised 27 August 2017; accepted 27 August 2017; posted 31 August 2017 (Doc. ID 297580); published 26 September 2017

\begin{abstract}
In this work, we investigate the superfluidic properties of light propagating in a four-level coherent atomic medium. The model is derived under the paraxial approximation in the form of a generalized nonlinear Schrödinger equation and features spatially controllable and quantum-enhanced optical properties, which can offer new possibilities in the field of optical analogue systems. In particular, we use this versatility to study the dynamics of an optical vortex beam confined in a nontrivial connected geometry, finding numerical evidence of another superfluidic signature analogue: the persistent current of light. @ 2017 Optical Society of America
\end{abstract}

OCIS codes: (270.1670) Coherent optical effects; (190.4420) Nonlinear optics, transverse effects in.

https://doi.org/10.1364/JOSAB.34.002220

\section{INTRODUCTION}

Optical analogues of many-body physical systems appear as a promising tool for exploring systems that span from general relativity [1-4] to quantum effects [5]. In these experiments, the effective interactions between photons are determined by the optical properties of matter, typically related with its nonlinear response [5]. Thus, the need to explore systems whose optical properties can be easily tailored is a problem of paramount importance.

The superfluidic behavior of light has received some attention in recent years [6]. Since the first observations of superconductivity and superfluidity, the exploration of these macroscopic quantum phenomena during the past decades took advantage from the development of a theoretical framework relating these effects with Bose-Einstein condensates (BECs) $[7,8]$. Indeed, BECs constitute a highly tunable platform that allow the control of a wide range of parameters, from the density to the interaction between atoms. As a result, superfluidic behavior has been extensively analyzed using BECs, first in ultracold atomic gases $[9,10]$ and more recently in excitonpolariton fluids [5,11-13]. However, the technological challenges involved in these experiments strongly limit their experimental implementation in configurations of increasing complexity. This paves the way for the development of alternative platforms to emulate of these effects.

The similarity between the model for monochromatic beam propagation in bulk nonlinear media under the paraxial approximation and the Gross-Pitaevskii equation describing the dynamics of a BEC allows the interpretation of light as a many-body physical system [6,14]. Furthermore, the wellknown hydrodynamic description of light identifies the intensity of light and the gradient of the phase of the optical field with the density and velocity of a fluid $[15,16]$. In this context, typical superfluidic signatures are predicted to occur below the critical speed defined by the Landau criterion [7,17]. Recently, there have been theoretical and experimental advances on this subject, including the study of dissipationless motion [18], suppressed scattering $[6,18-20]$, and the formation of shock wave diffraction patterns by a defect, with both dark solitons and vortices [21-24]. Still, the study of these quantum phenomena is limited when using bulk nonlinear media, which are not suitable to reproduce other superfluidic signatures, including the hallmark of superfluidity: the existence of persistent currents. Therefore, these optical media should be replaced by other materials that allow a spatial control of the linear and the nonlinear optical properties. Although photorefractive crystals can be used for that purpose [6,25], their relatively weak nonlinearity and the consequently large propagation distance needed to obtain these effects strongly limits the use of these materials.

In this context, quantum atomic optical systems such as atomic gases can constitute a good alternative. In these systems, quantum interference and atomic coherence between electronic levels can be exploited to develop highly tunable optical media with spatial control of both linear and nonlinear susceptibility using an external control beam [26,27], while strongly suppressing near-resonance absorption effects under the electromagnetically induced transparency regime [28]. Also, these can exhibit a large enhancement of the Kerr nonlinearity, even 
at low intensities (down to the few-photons regime) [28,29], making it an extremely appealing optical medium to study nonlinear phenomena, including the realization and control of spatial [26], vectorial [30], and temporal dissipative solitons [31].

In this paper, we consider an $N$-type four-level atomic system and use it to obtain a highly tunable optical medium suitable for the development of optical analogue simulations. We derive a generalized nonlinear Schrödinger equation governing the propagation of a probe optical field, featuring not only enhanced third-order nonlinearity but also allowing a spatial variation of the linear refractive index, induced by an external optical switching field. Introducing the hydrodynamic model for this light fluid, we derive the Bogoliubov dispersion relation for small perturbations on top of the light fluid created by a linear defect induced by the optical switching field and observe the characteristic superfluidic signature of suppressed scattering. Moreover, we explore the possibility of controlling the linear refractive index to confine light into a connected geometry and use this system to predict and observe numerically persistent currents in the light fluid, establishing a optical analogue of this well-known quantum phenomenon.

\section{MODEL}

This paper considers an $N$-type four-level atomic system interacting with three continuous-wave optical fields, as represented in Fig. 1. The levels $|1\rangle,|2\rangle$, and $|3\rangle$ constitute a typical three-level $\Lambda$ scheme, with a weak probe field $E_{p}=$ $\frac{1}{2}\left[\mathcal{E}_{p}(r, z) e^{i k_{p} z-i \omega_{p} t}+\right.$ c.c. $]$ and a strong control field $E_{c}=$ $\frac{1}{2}\left[\mathcal{E}_{c}(r) e^{i k_{p} z-i \omega_{p} t}+\right.$ c.c. $]$ driving the transitions $|1\rangle \rightarrow|3\rangle$ and $|2\rangle \rightarrow|3\rangle$ with envelope functions $\mathcal{E}_{p, c}(r)$, center frequencies $\omega_{p, c}$, and wave vectors $k_{p, c}$, respectively. Additionally, there is a supplementary strong switching field $E_{s}=$ $\frac{1}{2}\left[\mathcal{E}_{s}(r, z) e^{i k_{s} z-i \omega_{s} t}+\right.$ c.c. $]$ that coherently couples levels $|2\rangle$ and $|4\rangle$ at frequency $\omega_{s}$ and wave vector $k_{s}$. Using a classical description for the fields and neglecting the effects of the weak probe beam in the dynamics of both the switching and control fields, the optical beam propagates according to the wave equation under the paraxial approximation as

$$
i k_{p} \partial_{z} \mathcal{E}_{p}+\frac{1}{2} \nabla_{\perp}^{2} \mathcal{E}_{p}=\frac{1}{2 \varepsilon_{0} c^{2}} \partial_{t}^{2} P_{p},
$$

where $P_{p}$ is the polarization resulting from the interaction of the fields with the atomic medium, defined as $P_{p}=$ $\eta \mu_{31} \rho_{31} e^{i\left(k_{p} z-\omega_{p} t\right)}+$ c.c. with $\eta$ being the atomic density, $\mu_{i j}$ the dipole moment of the transition $|i\rangle \rightarrow|j\rangle$, and $\rho_{i j}$ the elements of the density-matrix operator $\rho$. To obtain an analytical expression for the polarization term, we address the dynamics of the atomic populations described by the master equation

$$
\dot{\rho}=\frac{i}{\hbar}[\rho, \hat{H}]-\frac{\hat{\Gamma}(\rho)}{2},
$$

with $H$ being the Hamiltonian of the system and given by

$$
\begin{aligned}
\hat{H}= & \sum_{i=1}^{4} \hbar \omega_{i}|i\rangle\langle i|-\frac{\hbar}{2}\left(\Omega_{p} e^{-i \omega_{p} t} \hat{\sigma}_{31}\right. \\
& \left.+\Omega_{c} e^{-i \omega_{c} t} \hat{\sigma}_{32}+\Omega_{s} e^{-i \omega_{s} t} \hat{\sigma}_{42}+\text { H.c. }\right),
\end{aligned}
$$
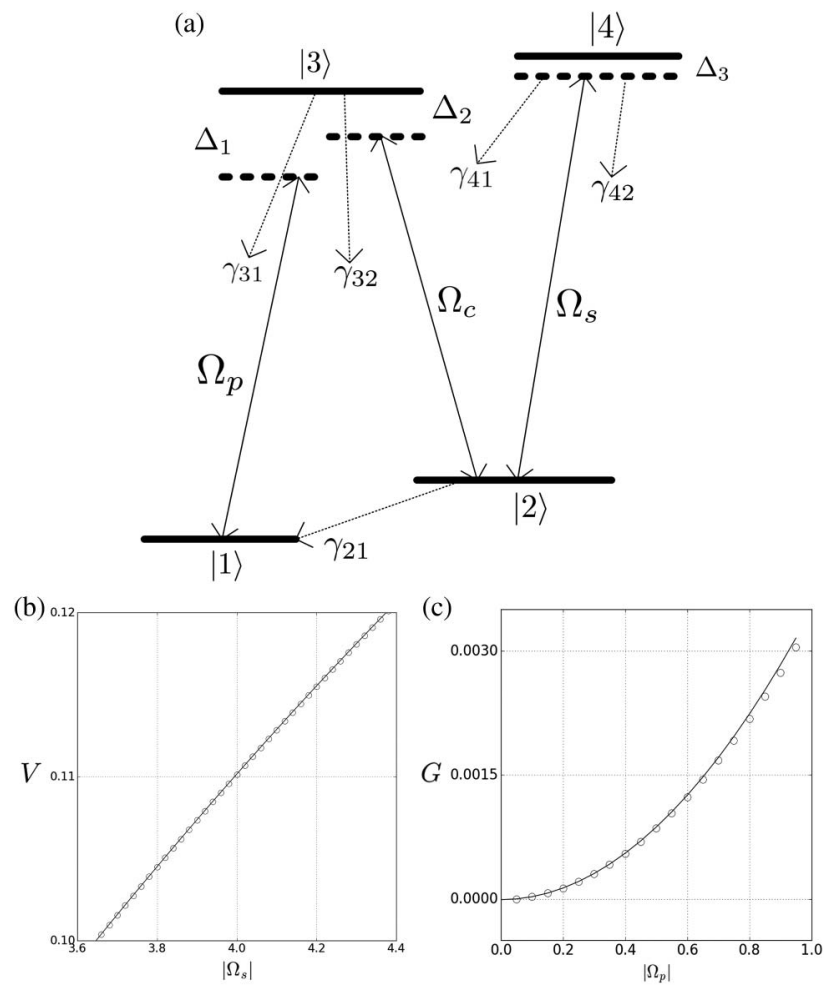

Fig. 1. (a) Schematic representation of the $N$-type four-level atomic system. A weak probe field drives the transition $|1\rangle \rightarrow|3\rangle$ with frequency $\omega_{p}$ and detuning $\Delta_{1}$ while two strong optical fields, a control and a switching field, couple $|2\rangle \rightarrow|3\rangle$ and $|2\rangle \rightarrow|4\rangle$ with frequencies $\omega_{c}$ and $\omega_{s}$ and detunings $\Delta_{2}$ and $\Delta_{3}$, respectively. Also included are the decoherence rates $\gamma_{i j}$, describing relaxation processes between two states. Comparison between the deduced simplified model (solid line) and the numerical results (circles) including losses for the real part of the susceptibility, both for the (b) linear term $V$ as a function of $\left|\Omega_{s}\right|$ and (c) the nonlinear term $G$ as a function of $\left|\Omega_{p}\right|$. For these results, we have used $\left|\Omega_{c}^{0}\right|=4$ and the detuning $\Delta=-30$, and in (c), we have additionally considered $\left|\Omega_{s}^{0}\right|=4$.

where the $\Omega_{i}$ are the Rabi frequencies for the transitions defined as $\Omega_{p}=\mu_{31} E_{p} / \hbar, \Omega_{c}=\mu_{32} E_{c} / \hbar$, and $\Omega_{s}=\mu_{42} E_{s} / \hbar$ and $\hat{\sigma}_{i j}=|i\rangle\langle j|$ is the atomic projection operator. We have also introduced the Lindblad superoperator $\hat{\Gamma}(\rho)$ that describes the relaxation and dephasing processes in our system. Using the above definitions, Eq. (1) becomes

$$
i \frac{1}{k_{p}} \partial_{z} \Omega_{p}+\frac{1}{2 k_{p}^{2}} \nabla_{\perp}^{2} \Omega_{p}+\chi_{p} \Omega_{p}=0,
$$

where the susceptibility is defined as

$$
\chi_{p}=\frac{\eta \mu_{31}^{2}}{\varepsilon_{0} \hbar \Omega_{p}} \rho_{31} \text {. }
$$

Assuming an instantaneous optical response, the equations describing the optical media and the field propagation can be decoupled by considering that the system attains a steady-state solution $\dot{\boldsymbol{\rho}}=0$. Neglecting the rapid oscillating terms by taking the rotating-wave approximation [32], Eq. (2) can be simplified [33] and expressed in a convenient matricial form as 


$$
\dot{\boldsymbol{\rho}}=\left(M_{0}+M_{p}\right) \boldsymbol{\rho},
$$

where $\rho$ is a vector containing the coefficients $\rho_{i j}$ of the density matrix and where $M_{0}$ and $M_{p}$ are matrices describing the $\Omega_{p}$-independent and -dependent parts of the dynamical equation for $\boldsymbol{\rho}$, respectively. Then, since both the control and the switching field are much stronger than the probe field (i.e., $\left.\left|\Omega_{p}\right| \ll\left|\Omega_{s}\right|,\left|\Omega_{c}\right|\right)$, the density-matrix elements can be obtained iteratively as $\rho_{i j}=\rho_{i j}^{(0)}+\rho_{i j}^{(1)}+\rho_{i j}^{(2)}+\rho_{i j}^{(3)}+\ldots$. Applying this expansion to Eq. (6), it is easy to obtain that the $n$th step of the density matrix should satisfy

$$
M_{0} \boldsymbol{\rho}^{(n)}=-M_{p} \boldsymbol{\rho}^{(n-1)} .
$$

Considering the ground state as the zeroth order solution (i.e., $\rho_{11}^{(0)}=1, \rho_{i j}^{(0)}=0$ if $i$ or $j \neq 1$ ), Eq. (7) yields

$$
\begin{aligned}
& \rho_{31}^{(1)}=A \Omega_{p}, \\
& \rho_{31}^{(2)}=0, \\
& \rho_{31}^{(3)}=B\left|\Omega_{p}\right|^{2} \Omega_{p},
\end{aligned}
$$

where the expressions for $A$ and $B$ are functions of the control and switching field amplitudes $\left|\Omega_{c}\right|$ and $\left|\Omega_{s}\right|$, the decoherence rates $\gamma_{i j}$ (accounting the relaxation and dephasing processes from state $|i\rangle$ to the state $|j\rangle)$. The single-photon detunings between the transition frequencies and those of the optical fields are given by $\Delta_{13}=\omega_{p}-\omega_{31}, \Delta_{23}=\omega_{c}-\omega_{32}$, and $\Delta_{42}=\omega_{d}-\omega_{42} \equiv \Delta_{2}$, with $\omega_{i j}=\omega_{i}-\omega_{j}$. While the full expressions for these quantities are too cumbersome to be written here, a simplified limit can still be obtained. Neglecting the dephasing processes between the two ground states and considering that $\gamma_{32}=\gamma_{31}=\gamma_{42}=\gamma_{41}=\gamma$ for sufficiently large and equal detunings, i.e., $\Delta_{1}=\Delta_{2}=\Delta \gg \gamma_{i j}$, $\Omega_{c, p}$, we obtain that

$$
\rho_{31} \approx-\frac{\left|\Omega_{s}\right|^{2}}{2 \Delta\left(\left|\Omega_{c}\right|^{2}+\left|\Omega_{s}\right|^{2}\right)} \Omega_{p}+\frac{\left|\Omega_{s}\right|^{2}}{2 \Delta\left(\left|\Omega_{c}\right|^{2}+\left|\Omega_{s}\right|^{2}\right)^{2}}\left|\Omega_{p}\right|^{2} \Omega_{p} .
$$

Finally, introducing the new variables $z^{\prime}=k_{p} z, x^{\prime}=k_{p} x$, $y^{\prime}=k_{p} y$, and $r^{\prime}=\sqrt{x^{\prime 2}+y^{\prime 2}}$, the transformation $\Omega_{p, s, c}^{\prime}=$ $\Omega_{p, s, c} / \gamma, \Delta_{1,2}^{\prime}=\Delta_{1,2} / \gamma$, and the coefficient $\kappa=\eta \mu_{31}^{2} /\left(\varepsilon_{0} \hbar \gamma\right)$, and dropping the primes, we obtain the dimensionless generalized nonlinear Schrödinger equation describing the evolution of the probe field as

$$
i \partial_{z} \Omega_{p}+\frac{1}{2} \nabla_{\perp}^{2} \Omega_{p}+V(r) \Omega_{p}-G(r)\left|\Omega_{p}\right|^{2} \Omega_{p}=0,
$$

where the effective linear coefficient is expressed as

$$
V(r)=-\frac{\kappa\left|\Omega_{s}\right|^{2}}{2 \Delta\left(\left|\Omega_{c}\right|^{2}+\left|\Omega_{s}\right|^{2}\right)},
$$

while the nonlinear term is associated with a self-Kerr effect and given by

$$
G(r)=-\frac{\kappa\left|\Omega_{s}\right|^{2}}{2 \Delta\left(\left|\Omega_{c}\right|^{2}+\left|\Omega_{s}\right|^{2}\right)^{2}} .
$$

The four-level scheme here considered can be implemented with a multitude of atomic systems. For an example, we can consider the hyperfine structure of the $\mathrm{D}$ line of ${ }^{87} \mathrm{Rb}$, assigning the levels $|1\rangle,|2\rangle,|3\rangle$, and $|4\rangle$ to the levels
$6 S_{1 / 2}(F=1), 6 S_{1 / 2}(F=2), 6 P_{1 / 2}(F=2)$, and $6 P_{3 / 2}(F=1)$, respectively. Using realistic physical parameters [34], the dipole matrix elements are $\mu_{13} \simeq 2.11 \times 10^{-29} \mathrm{Cm}, \mu_{23} \simeq$ $1.26 \times 10^{-29} \mathrm{Cm}$, and $\mu_{24} \simeq 1.79 \times 10^{-30} \mathrm{Cm}$ and the decay rates are $\gamma_{31}=\gamma_{32}=\gamma_{42}=\gamma_{41} \simeq \gamma$ and $\gamma_{21} \simeq 10^{-8} \gamma$ (with $\left.\gamma=36 \times 10^{6} \mathrm{~s}^{-1}\right)$. Also, we consider the wavelengths of the optical fields to be $\lambda_{p} \approx \lambda_{c}=795 \mathrm{~nm}$ and $\lambda_{s}=780 \mathrm{~nm}$ and consider a fixed atomic concentration $\eta=10^{15} \mathrm{~cm}^{-3}$.

Using these values and recovering the definition of Eq. (5), we plot the dependence of the real part of $\chi_{p}$ on the probe and switching field amplitudes for fixed detunings $\Delta_{1}=-30 \gamma$ and $\Delta_{2}=-30 \gamma$, computed numerically from the steady state of the density matrix. In Figs. 1(b) and 1(c), we observe a direct comparison between our model obtained from the perturbative approach and the numerical data including losses for both the linear and nonlinear terms of Eq. (10). Our model provides an excellent fit for relatively low values of $\Omega_{p}$ with small discrepancies occurring at larger values, as we breakaway from the weak-field assumption (i.e., $\left|\Omega_{p}\right| \ll\left|\Omega_{s}\right|,\left|\Omega_{c}\right|$ ) and correspond to higher-order nonlinearities [35,36]. Still, the relative error between our model and numerical calculations is below $3 \%$ for the parameter range considered here. We have also found numerically that $\operatorname{Re}\left\{\chi_{p}\right\} \lesssim 10^{2} \operatorname{Im}\left\{\chi_{p}\right\}$ for the physical parameters considered here, allowing us to safely neglect the losses for sufficiently small propagation distances [30,35].

The possibilities offered by this system to study the subject of light fluids are nontrivial, as it allows not only control of the strength of the nonlinear parameter but also provides a highly tunable spatial modulation of the linear refractive index. In particular, we are interested in the case of a spatially uniform control field $\left|\Omega_{c}(r)\right|=\left|\Omega_{c}^{0}\right|$ and a modulated switching field $\left|\Omega_{s}(r)\right|=\left|\Omega_{c}^{0}\right| \sqrt{1+f(r)}$, where $f(r)$ is a small spatially dependent perturbation. Expanding $V$ and $G$ to the first order in $f$, we obtain

$$
\begin{gathered}
V(r)=-\frac{\kappa}{4 \Delta}\left(1+\frac{f(r)}{2}\right), \\
G=-\frac{\kappa}{8 \Delta\left|\Omega_{c}^{0}\right|^{2}} .
\end{gathered}
$$

In the following section, we use this model to explore the superfluidic signatures in a fluid of light.

\section{SUPERFLUID LIGHT IN A FOUR-LEVEL ATOMIC MEDIUM}

First, we consider the scattering of a plane wave by a localized linear defect, a problem widely studied in the contexts of BECs and in the hydrodynamic interpretation of light $[5,6,11,12,37]$. Taking the Madelung representation

$$
\Omega_{p}=\left|\Omega_{p}^{0}\right| \mathrm{e}^{i \phi(x)},
$$

Equation (10) can be rewritten into two coupled hydrodynamic-like equations:

$$
\partial_{z} \rho+\nabla(\rho \boldsymbol{v})=0,
$$



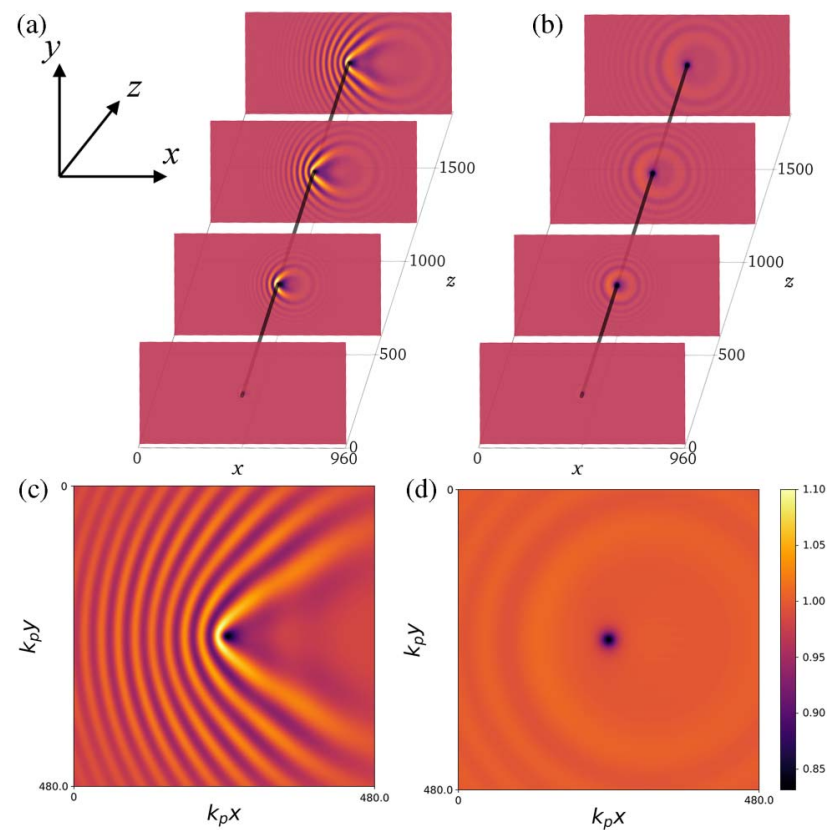

Fig. 2. Results for the numerical simulation of the scattering of a plane wave of initial condition $\Omega_{p}=\exp (i v x)$ by a defect, both in (a) the supersonic regime $v \approx 2.0 c_{s}$ and (b) the superfluid regime $v \approx 0.5 c_{s}$. The evolution of light fluid $\left|\Omega_{p}(z)\right|$ can be observed in the planes taken at increasing propagation distance $z$. The small defect, created by a Gaussian spatial profile with parameters $\delta=0.01$ and $\sigma=5$, is also represented schematically by the dark cylindrical surface. Details of the asymptotic transverse intensity profiles of the probe beam intensity $\left|\Omega_{p}\right|$ are presented both for (c) the supersonic regime and (d) the superfluid regime, for the same numerical simulations, taken at $k_{p} z=2500$.

$$
\partial_{z} \boldsymbol{v}+(\boldsymbol{v} \cdot \nabla) \boldsymbol{v}=-\nabla\left(V+G \rho+\frac{\nabla^{2} \rho^{1 / 2}}{\rho^{1 / 2}}\right)
$$

where the fluid velocity and density are defined as $v=\nabla \phi$ and $\rho=\left|\Omega_{p}^{0}\right|^{2}$, respectively. At this point, it is noteworthy that the density $\rho$ is proportional to the light intensity, while the velocity $v$ is associated with an incidence angle of the probe beam relative to the optical axis $z[6]$. According to this interpretation, we assume that at $z=0$, the probe field is given by a plane wave as

$$
\Omega_{p}(r, 0)=\left|\Omega_{p}^{0}\right| \mathrm{e}^{i v x},
$$

which corresponds to a fluid moving with velocity $v$, and that the switching field has a Gaussian-shaped defect on a constant background that can be expressed as

$$
f(r)=\delta \exp \left(-\frac{r^{2}}{2 \sigma^{2}}\right)
$$

Provided that the defect is relatively small, the scattering process results in elementary excitations with small amplitude on top of the initial state [38] that follow the well-known Bogoliubov dispersion relation

$$
\omega_{\text {Bog }}\left(\boldsymbol{k}_{\perp}\right)=\sqrt{k_{\perp}^{2}\left(\frac{k_{\perp}^{2}}{4}+G\left|\Omega_{p}^{0}\right|^{2}\right)} .
$$

In this framework, two regimes have been identified in the literature [6] depending on the value of the wavevector $k_{\perp}$ compared with the healing length $\xi=\sqrt{1 /\left(G\left|\Omega_{p}^{0}\right|^{2}\right)}$ : large momentum excitations (with $k_{\perp} \xi \gg 1$ ) follow a parabolic dispersion relation with $\omega_{\mathrm{Bog}}\left(\boldsymbol{k}_{\perp}\right) \approx \frac{k_{\perp}^{2}}{2}$, typical of singleparticle behavior, whereas small momentum excitations (with $\left.k_{\perp} \xi \ll 1\right)$ follow a sonic dispersion relation with $\omega_{\text {Bog }}\left(\boldsymbol{k}_{\perp}\right) \approx c_{s} k_{\perp}$, where $c_{s}=\sqrt{G\left|\Omega_{p}^{0}\right|^{2}}$ is the velocity of sound, typical of a collective response. Thus, and according to the Landau criterion, the light fluid behaves as a superfluid for sufficiently small velocities $v<c_{s}[6,7,17]$.

Figures 2(a) and 2(b) show the results of the numerical simulations for the values $v \approx 2.0 c_{s}$ and $v \approx 0.5 c_{s}$, respectively. The asymptotic behavior after the transient has passed are shown in Figs. 2(c) and 2(d). The occurrence of the two regimes described above can be identified: for flows at supersonic regime, we observe the characteristic Mach-Cerenkov cone [23], while for the sub-sonic regime, we notice very small fluctuations of the density upstream the defect that correspond to the frictionless flow of the light fluid around the defect $[5,6]$. For this simulation, we have used the parameters $\Delta=-30$ and $\left|\Omega_{c}^{0}\right|=$ $\left|\Omega_{s}^{0}\right|=4$ and for the defect we have chosen the values $\delta=$ 0.01 and $\sigma=5$. Also, we have considered the amplitude of the probe field $\left|\Omega_{p}^{0}\right|=1$. We have checked numerically that these results hold, even when considering the loss terms previously neglected, thus confirming numerically that this superfluidic signature could in principle be observed experimentally.

While the numerical results presented here were already predicted for other nonlinear materials [6,39], we foresee that the optical media here introduced could offer different experimental possibilities. In particular, using an atomic gas should allow us to observe an optomechanical signature of the superfluid flow of light as proposed in Ref. [19], both in obstacles and defects that can be embedded in the media or in the switching optical field itself, an idea that we will explore in a separate work.

Another interesting property of this medium is that it allow us to reach larger values for the nonlinear refractive index. For example, for the simulation considered in this section, we obtain a nonlinear refractive index of the order of $\Delta n \approx 3 \times 10^{-3}$, at least one order of magnitude larger than the optical media typically used $[6,19,39]$. As we are interested in the low-frequency modes associated with the sonic dispersion, we need to consider propagation distances sufficiently large to support a full period of oscillation for these modes, i.e., the minimum propagation distance should satisfy $z_{\min } \gg \lambda_{p} / \Delta n$ [39]. Consequently, higher nonlinear parameters reduce the propagation distance from the decimeter to the centimeter range, which can constitute an advantage in experimental characterization of the superfluidic behavior of light [39].

\section{SUPERFLUID PROPERTIES OF LIGHT IN CONNECTED GEOMETRIES}

When confined in connected geometries, the existence of persistent currents or supercurrents is a manifestation of the 
dissipationless flow of a superfluid across a defect. In this section, we will establish the optical analogue of this phenomenon by using the switching optical field to create a linear potential that can effectively trap an optical beam with orbital angular momentum in a connected geometry.

We assume that initially the intensity distribution for the probe beam can be approximated by

$$
\Omega_{p}(r, \theta)=\left|\Omega_{p}^{0}\right| \exp \left(-\frac{\left(r-r_{0}\right)^{2}}{w^{2}}\right) \exp (i l \theta),
$$

which corresponds to the expression of a perfect vortex beam in polar coordinates, a profile currently realizable experimentally [40]. Here, $r_{0}, w$, and $l$ are, respectively, the radius, the width, and the angular momentum of the beam. According to the hydrodynamic interpretation, Eq. (21) describes the flow of a light fluid in a connected geometry with velocity $v_{0} \equiv l / r_{0}$ and density $\rho_{0} \equiv\left|\Omega_{p}^{0}\right|^{2}$. Also, the intensity distribution of the switching field is

$$
f(r, \theta)=I \exp \left(-\frac{\left(r-r_{0}\right)^{2}}{w_{f}^{2}}\right)+U(r, \theta)
$$

which determines the shape of the linear trapping potential $V(r)$, according to Eq. (13). The first term in Eq. (22) describes a ring-shaped potential well of depth $I$ that confines the vortex beam, while the second term corresponds to a potential barrier, described by $U(r, \theta)$, that will be used to probe the supercurrent state. Following the approach proposed in Ref. [41], we consider that the barrier size is much larger than the healing length, allowing us to adopt the hydraulic approximation [41] and neglect the Bohm potential in Eq. (17). Taking $r=r_{0}$ and $z=0$, we obtain a simplified onedimensional model for the stationary flow of light in the form of a Bernoulli equation as

$$
\frac{1}{2} v(\theta)^{2}+G \rho(\theta)+U(\theta)=\frac{v_{0}^{2}}{2}+G \rho_{0},
$$

where $v_{0}$ and $\rho_{0}$ are, respectively, the velocity and the density sufficiently far away from the barrier, corresponding to initial conditions set by Eq. (21).

The barrier potential forces a decrease in the density (and consequently, of the velocity of sound), while the velocity of the fluid increases. According to the Landau criteria, if the velocity of sound drops below the velocity of the fluid (or equivalently, if the velocity of fluid exceeds the velocity of sound), the fluid of light enters a dissipative regime. This occurs when the barrier reaches a value of the potential $U_{\max }$ at which the velocity of sound coincides with the velocity of the fluid of light. Also, as we have an inhomogeneous radial density profile, a small correction factor to the sound speed should be taken into account $[42,43]$. Here, we use the reduction by a factor $\sqrt{2}$ predicted for harmonic channels. Then using the corrected sound velocity, we have that at $\theta_{b}$

$$
c_{s}^{\prime}\left(\theta_{b}\right)=\frac{c_{s}\left(\theta_{b}\right)}{\sqrt{2}}=\sqrt{\frac{G \rho\left(\theta_{b}\right)}{2}} .
$$

This defines a critical value for the velocity at the barrier [43], which, together with Eq. (23) and the continuity condition imposed by Eq. (16), can be used to obtain a threshold value for the barrier potential

$$
U_{\max }=\frac{v_{0}^{2}}{2}+G \rho_{0}-\frac{5}{4}\left(\sqrt{2} G \rho_{0} v_{0}\right)^{2 / 3} .
$$

The existence of persistent currents was investigated using numerical simulations that consider a perfect vortex probe beam with initial conditions $\left|\Omega_{p}^{0}\right|=1.0, r_{0}=600, w=40$, and $l=5$. We have also considered that $\left|\Omega_{s}^{0}\right|=\left|\Omega_{c}^{0}\right|=4$ and that $f$ has a depth $I=-0.1$, with the same radius of the probe beam and width $w_{f}=50$. The barrier has a Gaussian shape according to Eq. (19), centered at $r=r_{0}$ and $\theta=0$ and with parameter $\sigma=200$. The resulting linear trapping potential is schematically illustrated in Fig. 3. The results of the simulations are shown in Fig. 4 for $\delta_{1}=0.002$ [see Fig. 4(a)] and for $\delta_{2}=0.004$ [see Fig. 4(b)], corresponding to maximum values of $U(r)$ below and above the threshold value $U_{\max }$, respectively. Although the intensity plots in Figs. 4(a) and 4 (b) do not allow us to clearly identify the two regimes (the superfluid and dissipative regimes), it is possible to investigate them by analyzing the averaged current

$$
J \equiv \frac{1}{2 i} \int_{0}^{2 \pi}\left(\Omega_{p}^{*} \partial_{\theta} \Omega_{p}-\Omega_{p} \partial_{\theta} \Omega_{p}^{*}\right) \mathrm{d} r \mathrm{~d} \theta .
$$

The initial current at $z=0$ is $J=l N_{0}$, with $N_{0}$ given by $N_{0}=\int\left|\Omega_{p}(0)\right|^{2} \mathrm{~d} r \mathrm{~d} \theta$. For sufficiently long propagation distances and above the threshold value (dissipative regime), we should observe a decay of the current, whereas below the critical value (superfluid regime), the averaged current should be constant and remain equal to the initial value. This is confirmed by the numerical results presented in Fig. 4(c), where the persistent current can be observed when the maximum of the barrier is below $U_{\max }$ [see Fig. 4(c)(i)], in contrast to the decay observed above the threshold [see Fig. 4(c)(ii)].

Persistent currents are a manifestation of the superfluidic behavior of light associated with the defocusing nonlinearity. Indeed, the numerical results without the nonlinear term exhibit periodic oscillations, commonly observed in these

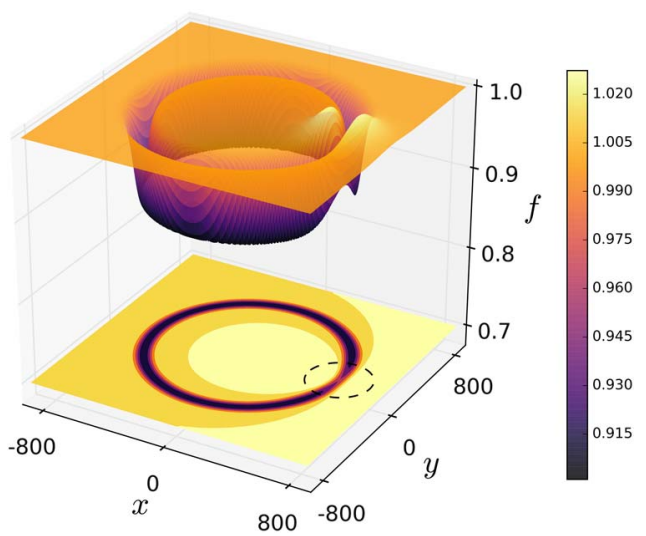

Fig. 3. Schematic illustration of the linear trapping potential used in the simulations where it is possible to identify the ring-like potential as well as the local barrier potential (dashed line). In this figure, the height of the Gaussian-shaped barrier was exaggerated tenfold to highlight the distortion imposed by the barrier on the ring potential. 
(a)

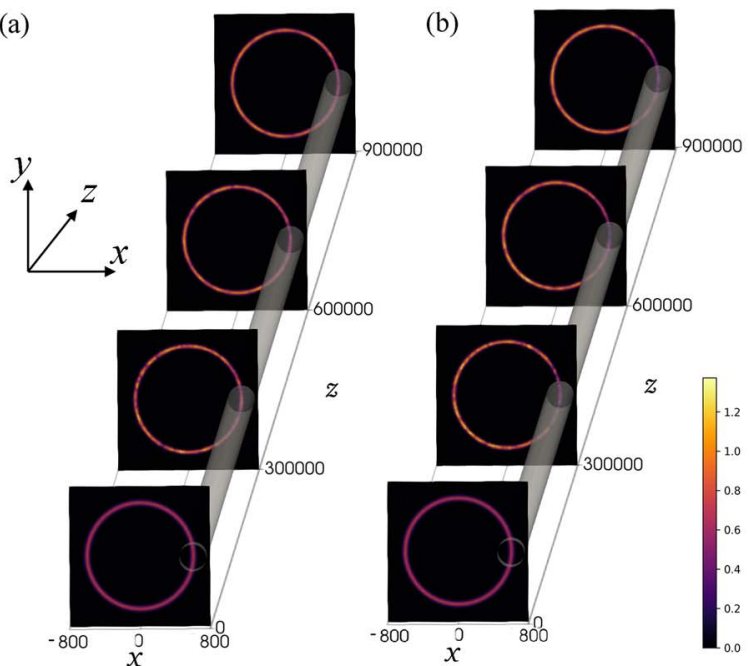

(c)

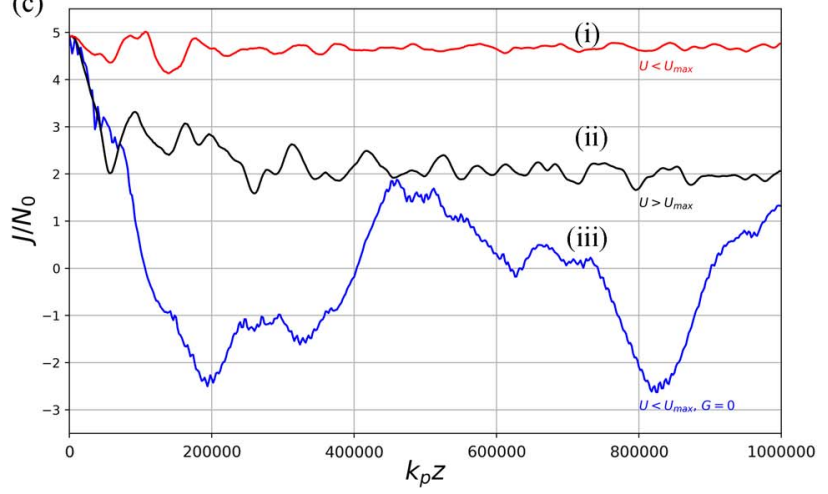

Fig. 4. Results for the numerical simulation of the evolution of a perfect vortex beam with initial conditions $\left|\Omega_{p}^{0}\right|=1.0, r_{0}=600$, $w=40$, and $l=5$, interacting with a potential barrier created by a Gaussian spatial profile with $\sigma=200$ and values (a) $\delta_{1}=0.002$ and (b) $\delta_{2}=0.004$, values that correspond to the maximum value of the potential barrier $U$ (a) below and (b) above the critical $U_{\max }$ value. The evolution of light fluid density $\left|\Omega_{p}(z)\right|$ can be observed in the planes taken at increasing propagation distance $z$ and the potential barrier is schematically represented by the gray cylindrical surface. In (c), we observe the dynamics of the averaged current $J$ for values of (i) (red line) $\delta_{1}=0.002$ and (ii) (dark line) $\delta_{2}=0.004$. Also represented is the result of a numerical simulation performed without the nonlinear term (iii) (blue line) for $\delta_{1}=0.002$.

systems [44] and associated with the Cherenkov synchronism between the obstacle and the fluid [Fig. 4(c)(iii)]. This contrasts with the case with the nonlinear terms that unveils persistent currents that exhibit either no decay at all [see Fig. 4(c)(i)] or a quantized decay from a state with $l=5$ to another with $l=2$ [see Fig. 4(c)(ii)].

This phenomenology has been extensively studied in context of superfluids and BECs and is associated with the particular superfluidic properties of the system $[13,45,46]$. Within the context of our work and of the fluid of light as an analogue system, the conservation of the current in the presence of a barrier can be associated with a state of dissipationless flow of light, which is a characteristic signature of superfluid systems known as a persistent current.

\section{CONCLUSIONS}

In this work, we have proposed an $N$-type four-level atomic system to explore the superfluid properties of light. Following a perturbative approach, we derived an effective generalized nonlinear Schrödinger equation for the propagation of a weak probe optical beam. The effective model includes linear and nonlinear terms that can be spatially modulated through the choice of appropriate additional control and switching optical fields.

Focusing on the context of the superfluid properties of light, we have shown that the versatility of these systems can be used to extend beyond the already reported phenomena. Introducing the hydrodynamic description of light, we have investigated the dynamics of an optical vortex beam confined into a nontrivial connected geometry. We have observed numerically that the fluid of light undergoes a dissipationless motion, even in the presence of a potential barrier, which we found to be associated with a superfluidic regime of light, thus constituting evidence of an optical analogue to persistent currents.

In conclusion, atomic optical media constitute a nontrivial addition to the field of optical analogue, especially due to its unique tunable optical properties. This can present new opportunities, allowing the realization of experimental setups that extend beyond the capabilities of other media previously considered. Furthermore, these media offer enhanced nonlinear parameters that reduce the propagation distances usually required to access the low-frequency modes, at which the superfluidic signatures occur. Finally, it is also noteworthy that this study can be extended beyond the effects explored in this paper. We foresee that interesting results could be observed in the nonperturbative approach, as established in Ref. [35], either in the dynamics of the probe beam itself, due to the large quintic nonlinear properties of the optical media, or in the mutual interaction between the probe, the control, and the switching fields. All this versatility could in principle be used to establish new directions of research and support further analogies between light fluids and many-body quantum systems.

Funding. Operational Programme for Competitiveness and Internationalisation-COMPETE 2020 Programme, European Regional Development Fund (ERDF); Fundação para a Ciência e a Tecnologia (FCT) (POCI-01-0145-FEDER006961, SFRH/BD/105486/2014).

\section{REFERENCES}

1. D. Faccio, T. Arane, M. Lamperti, and U. Leonhardt, "Optical black hole lasers," Classical Quantum Gravity 29, 224009 (2012).

2. T. G. Philbin, C. Kuklewicz, S. Robertson, S. Hill, F. König, and U. Leonhardt, "Fiber-optical analog of the event horizon," Science 319, 1367-1370 (2008).

3. F. Marino, "Acoustic black holes in a two-dimensional photon fluid," Phys. Rev. A 78, 063804 (2008).

4. D. Faccio, F. Belgiorno, S. Cacciatori, V. Gorini, S. Liberati, and U. Moschella, Analogue Gravity Phenomenology: Analogue Spacetimes and Horizons, from Theory to Experiment (Springer, 2013), Vol. 870.

5. I. Carusotto and C. Ciuti, "Quantum fluids of light," Rev. Mod. Phys. 85, 299-366 (2013) 
6. I. Carusotto, "Superfluid light in bulk nonlinear media," Proc. R. Soc. London A 470, 20140320 (2014).

7. L. Pitaevskii and S. Stringari, Bose-Einstein Condensation and Superfluidity (Oxford University, 2016), Vol. 164.

8. M. Greiner, C. A. Regal, and D. S. Jin, "Emergence of a molecular Bose-Einstein condensate from a Fermi gas," Nature 426, 537-540 (2003).

9. D. Butts and D. Rokhsar, "Predicted signatures of rotating BoseEinstein condensates," Nature 397, 327-329 (1999).

10. M. R. Matthews, B. P. Anderson, P. Haljan, D. Hall, C. Wieman, and E. Cornell, "Vortices in a Bose-Einstein condensate," Phys. Rev. Lett. 83, 2498-2501 (1999).

11. A. Amo, J. Lefrère, S. Pigeon, C. Adrados, C. Ciuti, I. Carusotto, R. Houdré, E. Giacobino, and A. Bramati, "Superfluidity of polaritons in semiconductor microcavities," Nat. Phys. 5, 805-810 (2009).

12. A. Amo, S. Pigeon, D. Sanvitto, V. Sala, R. Hivet, I. Carusotto, F. Pisanello, G. Leménager, R. Houdré, E. Giacobino, C. Ciuti, and A. Bramati, "Polariton superfluids reveal quantum hydrodynamic solitons," Science 332, 1167-1170 (2011).

13. D. Sanvitto, F. Marchetti, M. Szymańska, G. Tosi, M. Baudisch, F. Laussy, D. Krizhanovskii, M. Skolnick, L. Marrucci, A. Lemaitre, J. Bloch, C. Tejedor, and L. Viña, "Persistent currents and quantized vortices in a polariton superfluid," Nat. Phys. 6, 527-533 (2010).

14. P.-É. Larré and I. Carusotto, "Propagation of a quantum fluid of light in a cavityless nonlinear optical medium: general theory and response to quantum quenches," Phys. Rev. A 92, 043802 (2015).

15. E. Madelung, "Quantentheorie in hydrodynamischer form," Z. Phys. A 40, 322-326 (1927).

16. M. Vaupel, K. Staliunas, and C. Weiss, "Hydrodynamic phenomena in laser physics: modes with flow and vortices behind an obstacle in an optical channel," Phys. Rev. A 54, 880-892 (1996).

17. R. Y. Chiao and J. Boyce, "Bogoliubov dispersion relation and the possibility of superfluidity for weakly interacting photons in a twodimensional photon fluid," Phys. Rev. A 60, 4114-4121 (1999).

18. P. Leboeuf and S. Moulieras, "Superfluid motion of light," Phys. Rev. Lett. 105, 163904 (2010).

19. P.-É. Larré and I. Carusotto, "Optomechanical signature of a frictionless flow of superfluid light," Phys. Rev. A 91, 053809 (2015).

20. S. Batz and U. Peschel, "Superfluid motion of light past a solitonic obstacle," Phys. Rev. A 85, 033824 (2012).

21. W. Wan, S. Jia, and J. W. Fleischer, "Dispersive superfluid-like shock waves in nonlinear optics," Nat. Phys. 3, 46-51 (2007).

22. S. Jia, W. Wan, and J. W. Fleischer, "Dispersive shock waves in nonlinear arrays," Phys. Rev. Lett. 99, 223901 (2007).

23. E. G. Khamis, A. Gammal, G. El, Y. G. Gladush, and A. Kamchatnov, "Nonlinear diffraction of light beams propagating in photorefractive media with embedded reflecting wire," Phys. Rev. A 78, 013829 (2008).

24. E. Cohen, S. Muenzel, O. Farberovich, J. Fleischer, V. Fleurov, and A. Soffer, "Jet like tunneling from a trapped vortex," Phys. Rev. A 88 , 043833 (2013).

25. S. Jia and J. W. Fleischer, "Nonlinear light propagation in rotating waveguide arrays," Phys. Rev. A 79, 041804 (2009).

26. C. Hang and V. Konotop, "Spatial solitons in a three-level atomic medium supported by a Laguerre-Gaussian control beam," Phys. Rev. A 83, 053845 (2011).
27. Y. Li, B. A. Malomed, M. Feng, and J. Zhou, "Arrayed and checkerboard optical waveguides controlled by the electromagnetically induced transparency," Phys. Rev. A 82, 063813 (2010).

28. M. Fleischhauer, A. Imamoglu, and J. P. Marangos, "Electromagnetically induced transparency: optics in coherent media," Rev. Mod. Phys. 77, 633-673 (2005).

29. H. Wang, D. Goorskey, and M. Xiao, "Enhanced Kerr nonlinearity via atomic coherence in a three-level atomic system," Phys. Rev. Lett. 87, 073601 (2001).

30. Y. Qi, F. Zhou, T. Huang, Y. Niu, and S. Gong, "Spatial vector solitons in a four-level tripod-type atomic system," Phys. Rev. A 84, 023814 (2011).

31. M. Facão, S. Rodrigues, and M. Carvalho, "Temporal dissipative solitons in a three-level atomic medium confined in a photonic-band-gap fiber," Phys. Rev. A 91, 013828 (2015).

32. M. O. Scully and M. S. Zubairy, Quantum Optics (Cambridge University, 1997).

33. J. Sheng, X. Yang, H. Wu, and M. Xiao, "Modified self-Kerrnonlinearity in a four-level N-type atomic system," Phys. Rev. A 84, 053820 (2011).

34. D. A. Steck, "Rubidium $87 \mathrm{D}$ line data," http://steck.us/alkalidata.

35. A. Alexandrescu, H. Michinel, and V. M. Pérez-García, "Liquid like dynamics of optical beams in tailored coherent media," Phys. Rev. A 79, 013833 (2009).

36. H. Michinel, M. J. Paz-Alonso, and V. M. Pérez-García, "Turning light into a liquid via atomic coherence," Phys. Rev. Lett. 96, 023903 (2006).

37. I. Carusotto, S. Hu, L. Collins, and A. Smerzi, "Bogoliubov-Čerenkov radiation in a Bose-Einstein condensate flowing against an obstacle," Phys. Rev. Lett. 97, 260403 (2006).

38. J. T. Mendonça and H. Terças, Physics of Ultra-Cold Matter: Atomic Clouds, Bose-Einstein Condensates and Rydberg Plasmas (Springer, 2012), Vol. 70

39. D. Vocke, T. Roger, F. Marino, E. M. Wright, I. Carusotto, M. Clerici, and D. Faccio, "Experimental characterization of nonlocal photon fluids," Optica 2, 484-490 (2015).

40. M. Jabir, N. A. Chaitanya, A. Aadhi, and G. Samanta, "Generation of "perfect" vortex of variable size and its effect in angular spectrum of the down-converted photons," Sci. Rep. 6, 21877 (2016).

41. A. M. Leszczyszyn, G. El, Y. G. Gladush, and A. Kamchatnov, "Transcritical flow of a Bose-Einstein condensate through a penetrable barrier," Phys. Rev. A 79, 063608 (2009).

42. S. Stringari, "Dynamics of Bose-Einstein condensed gases in highly deformed traps," Phys. Rev. A 58, 2385-2388 (1998).

43. K. Wright, R. Blakestad, C. Lobb, W. Phillips, and G. Campbell, "Threshold for creating excitations in a stirred superfluid ring," Phys. Rev. A 88, 063633 (2013).

44. A. V. Yulin, Y. V. Bludov, V. V. Konotop, V. Kuzmiak, and M. Salerno, "Superfluidity of Bose-Einstein condensates in toroidal traps with nonlinear lattices," Phys. Rev. A 84, 063638 (2011).

45. A. Ramanathan, K. Wright, S. Muniz, M. Zelan, W. Hill III, C. Lobb, K. Helmerson, W. Phillips, and G. Campbell, "Superflow in a toroidal Bose-Einstein condensate: an atom circuit with a tunable weak link," Phys. Rev. Lett. 106, 130401 (2011).

46. S. Moulder, S. Beattie, R. P. Smith, N. Tammuz, and Z. Hadzibabic, "Quantized supercurrent decay in an annular Bose-Einstein condensate," Phys. Rev. A 86, 013629 (2012). 